\title{
INTERNAL TREATMENT OF PROCESS WATERS IN PAPER PRODUCTION BY DISSOLVED AIR FLOTATION WITH NEW DEVELOPED CHEMICALS. PART 2: FIELD TRIALS
}

\author{
Ruben Miranda, Carlos Negro, Angeles Blanco* \\ Department of Chemical Engineering, Faculty of Chemistry, Complutense University of \\ Madrid - Avda. Complutense s/n 28040 Madrid (Spain)
}

\begin{abstract}
New chemicals, based on the synergistic effect between inorganic polyaluminium salts and organic cationic polyelectrolytes, have been tested previously at lab scale and the most efficient product has been selected for an industrial trial for a dissolved air flotation unit of a paper mill based on 100\% recovered paper (Part 1 of this work). A polyaluminium nitrate sulphate salt combined with a quaternary polyamine has been evaluated in a long-term industrial trial for assessing its potential benefits. Results indicate that this new chemical is able to improve the removal of contaminants by dissolved air flotation, especially when the contamination load of the waters is high, allowing a further closure of the water circuits.
\end{abstract}

KEYWORDS: chemical synergistic effects, dissolved air flotation, flocculation, aluminium salts, polyamines, paper recycling, field trial.

\section{INTRODUCTION}

Nowadays, the destabilization and removal of recalcitrant contaminants, mainly dissolved and colloidal material (DCM), is still a bottleneck for papermakers ${ }^{1-2}$. DCM may be removed by dissolved air flotation (DAF) units if their chemistry is optimized ${ }^{3-}$ ${ }^{5}$. Part 1 of this work analyzes the chemical efficiency of newly developed chemicals for treating deinking process waters by $\mathrm{DAF}^{6}$. The new chemicals consider the potential synergistic effect between inorganic and organic products. Three types of aluminium salts modified with three different cationic polyelectrolytes were evaluated. The aluminium salts are: polyaluminium chloride, polyaluminium chloride sulphate and polyaluminium nitrate sulphate; and the cationic polyelectrolytes are polyacrylamide, polyvinylamide and polyamine. A total of thirteen products were analyzed at lab scale: the three aluminium salts of base and ten modifications of these salts.

\footnotetext{
${ }^{*}$ To whom correspondence should be addressed. Dpt. Chemical Engineering, Faculty of Chemistry, Complutense University of Madrid. Avda. Complutense s/n E-28040 Madrid (Spain). Phone: +34913944247. Fax: +34913944243. E-mail: ablanco@quim.ucm.es
} 
The efficiency of the different treatments was determined by comparing the contamination load of clarified waters and their DCM fraction (obtained by centrifugation) before and after flotation in a lab-scale DAF unit. Products were evaluated at their individual optimal dosage, determined by Focused Beam Reflectance Measurement (FBRM), and at a similar dosage for all (700-800 mg/L). These tests were carried out with waters from newsprint production. Results indicated that polyamine modifications significantly improved the efficiency of the aluminium salts. Removal efficiencies were the following: $90 \%$ of turbidity before centrifugation and $50 \%$ after centrifugation, $70 \%$ in the cationic demand (before and after centrifugation) and around $15 \%$ of the COD after centrifugation. Chemical dosages were relatively high compared to normal industrial dosages due to the higher hydrodynamic efficiency of industrial units and the accumulation of the chemicals in the water circuits.

According to these results, the modification of the polyaluminium nitrate sulfate with polyamine (PANS-PA) was selected for further studies. Results showed that: a) intermediate dosages are sufficient for high removal efficiencies of turbidity while removal efficiencies of cationic demand and COD are linearly related to the dosage of PANS-PA; b) great benefits are obtained using PANS-PA in combination with a flocculant (dual system); c) efficiency of PANS-PA is better for the removal of DCM compared to reference products used at the mill if dual systems are used; d) PANS-PA is effective for the removal of contaminants for both newsprint and LWC process waters. However, due to the higher contamination load of LWC waters, higher dosages are necessary and the removal efficiencies obtained for newsprint waters are not achievable.

Therefore, the objective of Part 2 of this work is to evaluate, in a long-term field trial, the potential benefits of using PANS-PA for the treatment of process water by DAF in two different conditions: newsprint and LWC paper production, both in single and dual systems.

\section{MATERIALS AND METHODS}

\subsection{Description of the paper mill}

Field trials were carried out in a Spanish paper mill using 100\% recovered paper as raw material. The mill manufactures both newsprint paper and light weight coated (LWC) paper in the same line. The deinking line, installed in 1998, produces around 500 tons per day of pulp from a mixture of old newsprint (ONP), old magazines (OMG), and office paper (OP) with an average composition of $60 \%, 30 \%$ and $10 \%$, respectively. Figure 1 shows a simplified diagram of the deinking line with the corresponding water loops and DAF units. 


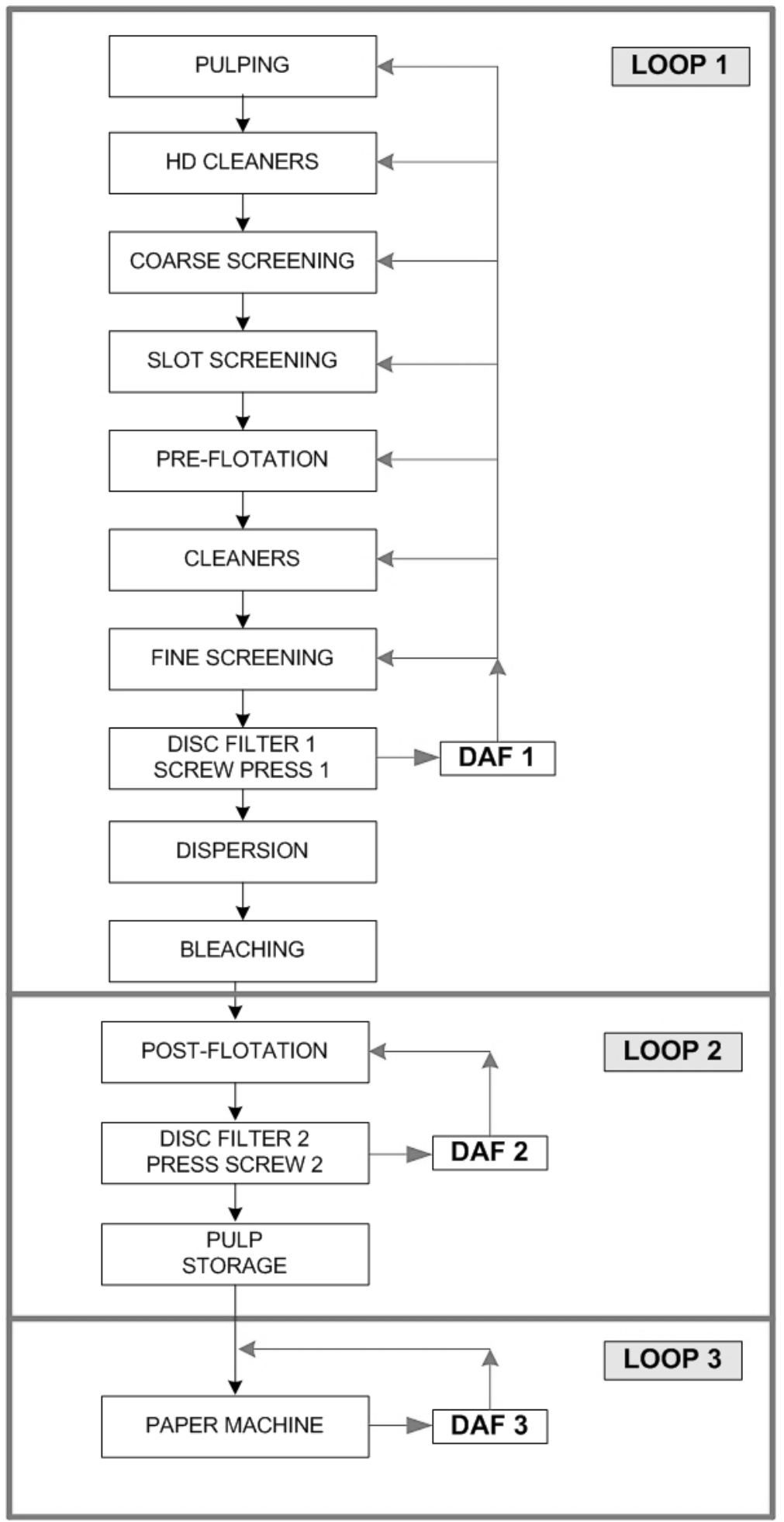

Figure 1. Simplified flow diagram of deinking line.

\subsection{Description of DAF unit}

Field trials were carried out in DAF 2, which treats the clear filtrate of the disc filter of loop 2. This unit treats a feed flow of between 2500 and $5000 \mathrm{~L} / \mathrm{min}$. It is a Deltapurge model unit, supplied by Meri, with circular geometry and following dimensions: $9.5 \mathrm{~m}$ of diameter and $1.5 \mathrm{~m}$ of height. Residence time is in the range $20-40$ 
min, depending on the inlet flow. PANS-PA (first) and flocculant (second), were added directly to the inlet waters, just before the pump feeding the inlet waters to DAF, in turbulent conditions and with a very short contact time (a few seconds).

\subsection{Description of PANS-PA}

The main properties of polyaluminium nitrate sulphate (PANS), with formula $\left[\mathrm{Al}(\mathrm{OH})_{\mathrm{x}}\left(\mathrm{NO}_{3}\right)_{\mathrm{y}}\left(\mathrm{SO}_{4}\right)_{\mathrm{z}}\right]_{\mathrm{n}}$, are summarized in Table 1 . This aluminium salt has been modified with a cationic quaternary polyamine with a $17 \%$ charge density for obtaining the PANS-PA product, which has a 1.8 wt. $\%$ content of cationic polyamine, a $3.2 \% \mathrm{Al}$ content and a dry content of $20.4 \%$. These products have been supplied by Sachtleben Chemie GmbH.

Table 1. Properties of the polyaluminium nitrate sulphate salt.

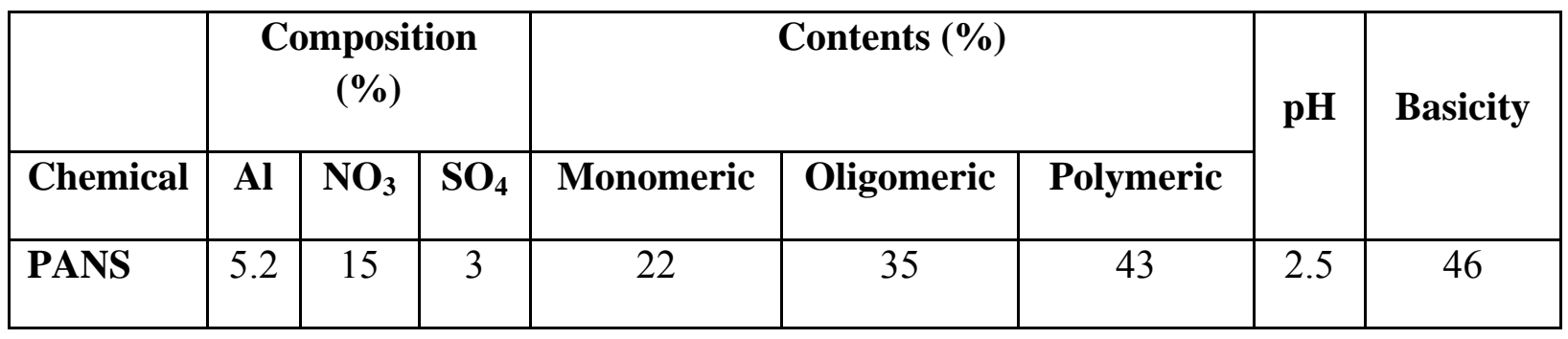

The modification of the aluminium salt with the polyamine induced an improvement in the flocculation of contaminants, especially those in the DCM fraction, which are the most difficult to remove in DAF systems. Figure 2 shows the main differences between the flocculation induced by the unmodified aluminium salt and its modification through the analysis of the chord size distribution of a sample treated at lab-scale with different dosages of both products. Flocculation with PANS increased the mean chord size of the raw waters from $13.9 \mu \mathrm{m}$ to $15.3 \mu \mathrm{m}(1000 \mathrm{mg} / \mathrm{L}), 16.7 \mu \mathrm{m}(2000 \mathrm{mg} / \mathrm{L})$ and $16.6 \mu \mathrm{m}$ $(3000 \mathrm{mg} / \mathrm{L})$ while, at the same time, the total number of counts decreased from 6800 \#/s (raw waters) to 5498 \#/s (1000 mg/L), 4537 \#/s (2000 mg/L) and 3957 \#/s (3000 $\mathrm{mg} / \mathrm{L})$. The analysis of the number of particles in the different chord size intervals shows that PANS induced the flocculation of particles between 1 and $100 \mu \mathrm{m}$ to produce larger flocs, easily removable by DAF systems. Flocculation with PANS-PA is different. First, a higher mean chord size of the particles is obtained with lower dosages of the product: the mean chord size of the particles increased from $13.9 \mu \mathrm{m}$ (raw waters) to $15.5 \mu \mathrm{m}(300 \mathrm{mg} / \mathrm{L}), 17.3(500 \mathrm{mg} / \mathrm{L})$ and $18.9 \mu \mathrm{m}(1000 \mathrm{mg} / \mathrm{L})$. Second, there is a continuous increase of the total number of particles at the same time than flocculation occurs, especially at low and medium dosages (from 100 to $500 \mathrm{mg} / \mathrm{L}$ ). The total number of counts increased from $6800 \mathrm{\#} / \mathrm{s}$ (raw waters) to $8730 \mathrm{\#} / \mathrm{s}(300 \mathrm{mg} / \mathrm{L}), 11802$ \#/s $(500 \mathrm{mg} / \mathrm{L})$ and 11204 \#/s $(1000 \mathrm{mg} / \mathrm{L})$. The increase in the mean chord size implies that particles in the range of $1-100 \mu \mathrm{m}$ are being flocculated and the continuous increase of the number of particles is due to the flocculation of particles smaller than $1 \mu \mathrm{m}$, which were not detected previously due to the $1-\mu \mathrm{m}$ detection limit of FBRM technique. 
As it can be seen, at $1000 \mathrm{mg} / \mathrm{L}$, the total number of particles starts to decrease smoothly, indicating that no more DCM is being destabilized; the increase in the mean chord size observed is due to the flocculation of the previously destabilized DCM.

Regarding to the flocculation mechanisms involved, the polyaluminium coagulants can act by two primary coagulation mechanisms ${ }^{7}$ : (1) charge neutralization of the negative charged particles by adsorption of positively charged dissolved aluminium species and (2) enmeshment of colloids in precipitated $\mathrm{Al}(\mathrm{OH})_{3}$ solids. Considering the $\mathrm{pH}$ of the waters to treat, between 7.5 and 8.5 , the main coagulation mechanism occurring is charge neutralization. In this flocculation mechanism, cations react chemically with negatively charged particles, partially or totally neutralising the charge of the particles and coagulation takes place at the iso-electric point of the system. This is the mechanism exhibited by the unmodified aluminium salt.

On the other hand, patch charge model is typical for cationic polyelectrolytes of low or medium molecular weight $(<100,000)$ and high charge density $(>4 \mathrm{meq} / \mathrm{g})$ as polyamines when added to suspensions of negatively charged particles. In this case, the polymer particles are completely adsorbed onto the particle surface, forming patches of positive charge. The patch formed changes the surface charge of the particle in the area where it is adsorbed, while the rest of the particle retains its initial charge. One the electrolyte has been adsorbed, the positively charged patches are attracted by negatively charged regions on the surface of other particles, so that the effect of this electrostatic attraction enhances the action of the van der Waals forces, causing the agglomeration of particles. Flocculation by this mechanism need not take place at the iso-electric point of the system, since the interaction is heavily depending on the charge of the local patches.

The addition of polyamine to the aluminium salts modified its flocculation behaviour in terms of a higher floc size, a higher coagulation of dissolved and colloidal material, a lower optimal dosage and a faster flocculation kinetic, which can be due to the evolution from a neutralization mechanism towards a partial patch flocculation mechanism. 


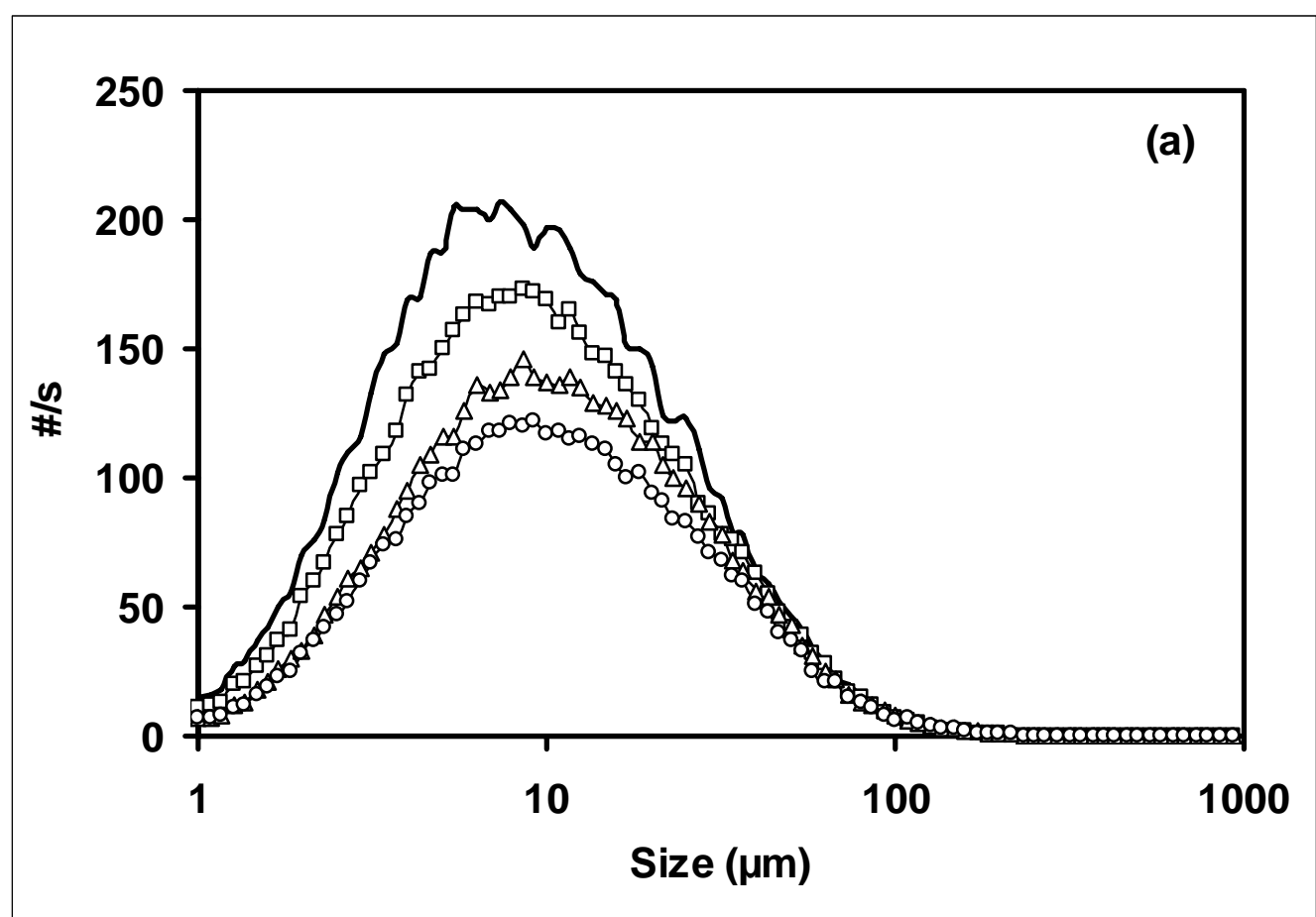

$\multimap 0 \mathrm{mg} / \mathrm{L} \rightarrow-1000 \mathrm{mg} / \mathrm{L} \quad \triangle-2000 \mathrm{mg} / \mathrm{L} \quad \multimap 3000 \mathrm{mg} / \mathrm{L}$

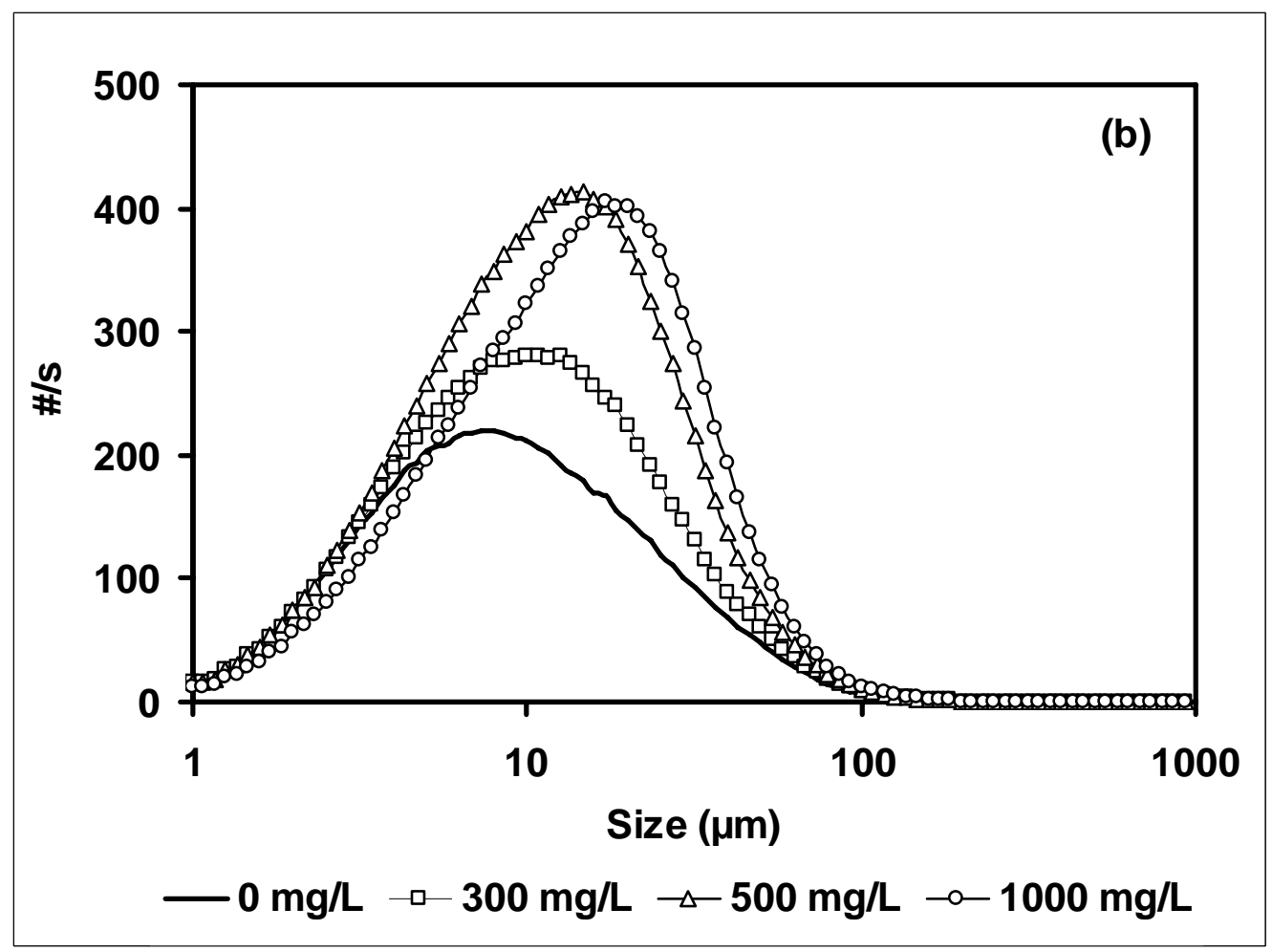

Figure 2. Chord size distribution curves obtained by the flocculation of a water sample (newsprint paper) with different dosages of (a) PANS and (b) PANS-PA. 


\subsection{Methodology}

First, a one-day preliminary trial has been carried out to detect possible problems during the substitution of the chemical used in the DAF. The operational conditions were: LWC paper production, inlet flow $=2500 \mathrm{~L} / \mathrm{min}$ and a flocculant dosage fixed to $3.6 \mathrm{mg} / \mathrm{L}$. Coagulant dosage was varied from $300 \mathrm{mg} / \mathrm{L}$, the optimal dosage obtained at lab-scale, to $100 \mathrm{mg} / \mathrm{L}$.

Second, DAF performance was studied during a long-term trial (53 days) for newsprint and LWC paper production, according to the calendar summarized in Figure 3. Newsprint production was studied during a total of 47 days: 8 days in stage A (before the start of the field trial for the establishment of a baseline), 7 days in stage B (first seven days of trial, just before the change to LWC paper production), and 32 days in stage D (after 3 days of LWC production and until the next change to LWC paper). A total of 21 samples were taken. LWC paper production was studied during a total of 6 days, according to regular production planning (2-3 days of LWC production per month): 3 days in stage $\mathrm{C}$ and 3 days in stage E. During these six days of trial, a total of 7 samples were taken.

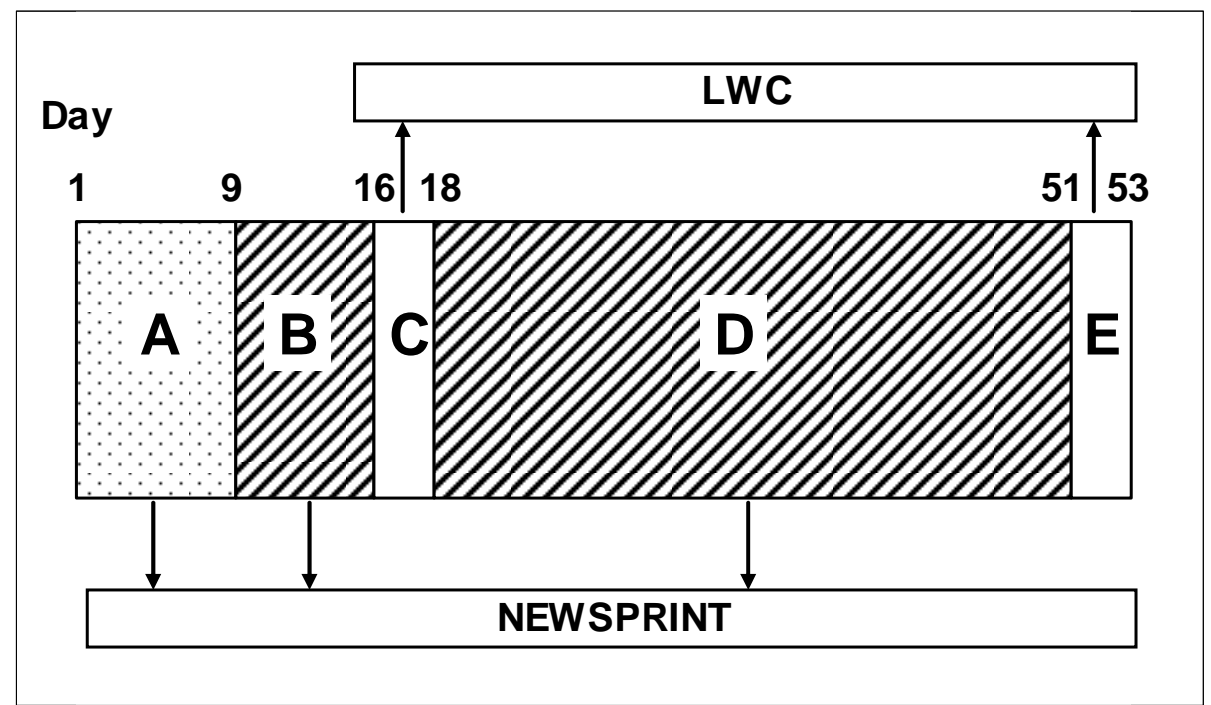

Figure 3. Chronogram of the long-term field trial. Stages (A through E) are defined in the text.

It is important to note these different stages during the trial because the contamination load of the waters is very different as shown in Table 2. For example, the contamination load in newsprint production just after LWC paper production is higher compared to regular newsprint paper production because of the higher contamination load of the circuits during LWC paper production. This is the case of the first days of stage $\mathrm{D}$, in which higher dosages of PANS-PA were equal or even less efficient than lower dosages of PANS-PA during the stage A or B. 
Table 2. Average values of the contamination load of the inlet waters to DAF during the field trial.

\begin{tabular}{|l|l|c|c|c|}
\hline Stage & $\begin{array}{l}\text { Paper } \\
\text { grade }\end{array}$ & $\begin{array}{c}\text { Conductivity } \\
\text { range (average) } \\
(\mathbf{m S / c m})\end{array}$ & $\begin{array}{c}\text { Cat. demand range } \\
\text { (average) }(\boldsymbol{\mu e q} / \mathbf{L})\end{array}$ & $\begin{array}{c}\text { COD range } \\
\text { (average) (ppm) }\end{array}$ \\
\hline $\mathbf{A}$ & $\begin{array}{l}\text { Newsprin } \\
\mathrm{t}\end{array}$ & $1.20 \rightarrow 1.40(1.30)$ & $\approx 400$ & $900 \rightarrow 1200(1050)$ \\
\hline $\mathbf{B}$ & $\begin{array}{l}\text { Newsprin } \\
\mathrm{t}\end{array}$ & $1.05 \rightarrow 1.45(1.25)$ & $300 \rightarrow 400(350)$ & $900 \rightarrow 1200(1050)$ \\
\hline $\mathbf{C}$ & $\mathrm{LWC}$ & $3.25 \rightarrow 4.0(3.75)$ & $2200 \rightarrow 3000(2600)$ & $2600 \rightarrow 3150(2850)$ \\
\hline $\mathbf{D}$ & $\begin{array}{l}\text { Newsprin } \\
\mathrm{t}\end{array}$ & $2.5 \rightarrow 1.4(1.95)$ & $600 \rightarrow 450(525)$ & $1900 \rightarrow 1400(1650)$ \\
\hline $\mathbf{E}$ & LWC & $3.75 \rightarrow 4.00(3.88)$ & $1700 \rightarrow 2600(2150)$ & $\approx 2900$ \\
\hline
\end{tabular}

Note: " $\rightarrow$ " indicates the contamination load tendency since the first days to the last days of each stage.

The removal efficiency of the DAF unit was determined in the following conditions: a) no addition of chemicals, b) addition of only flocculant, at a fixed dosage of $3 \mathrm{mg} / \mathrm{L}$ $(0 / 3), c)$ addition of only coagulant (dosages between 70 and $300 \mathrm{mg} / \mathrm{L})(70 / 0$ to 300/0) and d) combined addition of coagulant and flocculant, at $3 \mathrm{mg} / \mathrm{L}$ fixed dosage of flocculant and coagulant dosage varying from 70 to $300 \mathrm{mg} / \mathrm{L}$, e.g. 70/3 and 300/3.

Samples from both trials were characterized for turbidity, cationic demand, COD and TOC, following the same measurement methods as described in Part 1 of this work. Microstickies and secondary stickies (after destabilization of DCM with polyethilenimine) were also measured in the most representative samples as deposits collected in the UCM-deposition rotor $^{8-9}$.

\section{RESULTS AND DISCUSSION}

\subsection{One-day short trial}

First, a one-day trial was carried out to avoid possible problems in the substitution of the chemical used in DAF with the new product and for adjusting its dosage. The characterization of the feed waters and their DCM fraction is summarized in Table 3. 
Table 3. Characterization of feed waters during the short trial.

\begin{tabular}{|c|c|c|c|c|c|c|}
\hline Sample & $\mathbf{p H}$ & $\begin{array}{c}\text { Conductivity } \\
\mathbf{2 5}^{\mathbf{}} \mathbf{C} \\
\mathbf{( m S / c m )}\end{array}$ & $\begin{array}{c}\text { TS } \\
\mathbf{( g / L )}\end{array}$ & $\begin{array}{c}\text { Turbidity } \\
\mathbf{( N T U )}\end{array}$ & $\begin{array}{c}\text { CD } \\
\mathbf{( m e q} / \mathbf{L})\end{array}$ & $\begin{array}{c}\text { COD } \\
\mathbf{( p p m )}\end{array}$ \\
\hline Inlet Waters & 8.4 & 3.75 & 6.45 & 5200 & 1.69 & - \\
\hline DCM fraction & 8.4 & 3.75 & - & 63 & 1.67 & 2904 \\
\hline
\end{tabular}

First, a conservative dosage of $300 \mathrm{mg} / \mathrm{L}$ was tested. As the quality of the clarified waters was good enough, the dosage was reduced to $200 \mathrm{mg} / \mathrm{L}$ for a first period, and then to $100 \mathrm{mg} / \mathrm{L}$ for a second period. The main results obtained in this trial are summarized in Figure 4. If only flocculant is added, a 13\% removal of turbidity is achieved. But if PANS-PA is added with the same dosage of flocculant, the removal efficiency increases to $96 \%$ with $100 \mathrm{mg} / \mathrm{L}$ and to around $98 \%$ for 200 or $300 \mathrm{mg} / \mathrm{L}$. If we consider the DCM fraction, no removal of turbidity is achieved with only flocculant but with PANS-PA, turbidity can be removed up to $42 \%$, indicating that coagulant is completely necessary for the removal of contaminants in the DCM fraction. Little differences in turbidity after centrifugation for the different dosages of PAN-PA were observed. In the case of cationic demand, no reduction is observed if only flocculant is added (a $2 \%$ reduction). With PANS-PA, cationic demand can be removed by $20 \%$ (100 $\mathrm{mg} / \mathrm{L}), 30 \%(200 \mathrm{mg} / \mathrm{L})$ and $35 \%(300 \mathrm{mg} / \mathrm{L})$. Cationic demand removal is similar both before and after centrifugation, its reduction being almost linear with the PANS-PA dosage. The same occurred with COD and TOC. No reduction of COD or TOC was observed with flocculant addition; with increasing concentrations of PANS-PA small reductions are achieved: $2 \%$ with $100 \mathrm{ppm}, 4 \%$ with $200 \mathrm{ppm}$ and $6 \%$ with $300 \mathrm{ppm}$. Similar reductions of TOC were obtained (1-7\%).

As occurred in lab trials, PANS-PA dosage is important in terms of removal of cationic demand, COD and TOC. Differences in turbidity removals with different PANS-PA dosages are low. If only turbidity removal is necessary, dosages of $100 \mathrm{mg} / \mathrm{L}$ or even lower are sufficient. This could be considered the optimal dosage although it depends on the contamination load of the inlet waters. It is also confirmed that the use of flocculant alone is clearly not efficient for cleaning the process waters in LWC production. If the removal of contaminants in the DCM fraction is needed, mandatory for reducing fresh water consumption, destabilisation of DCM by a coagulant is necessary. 

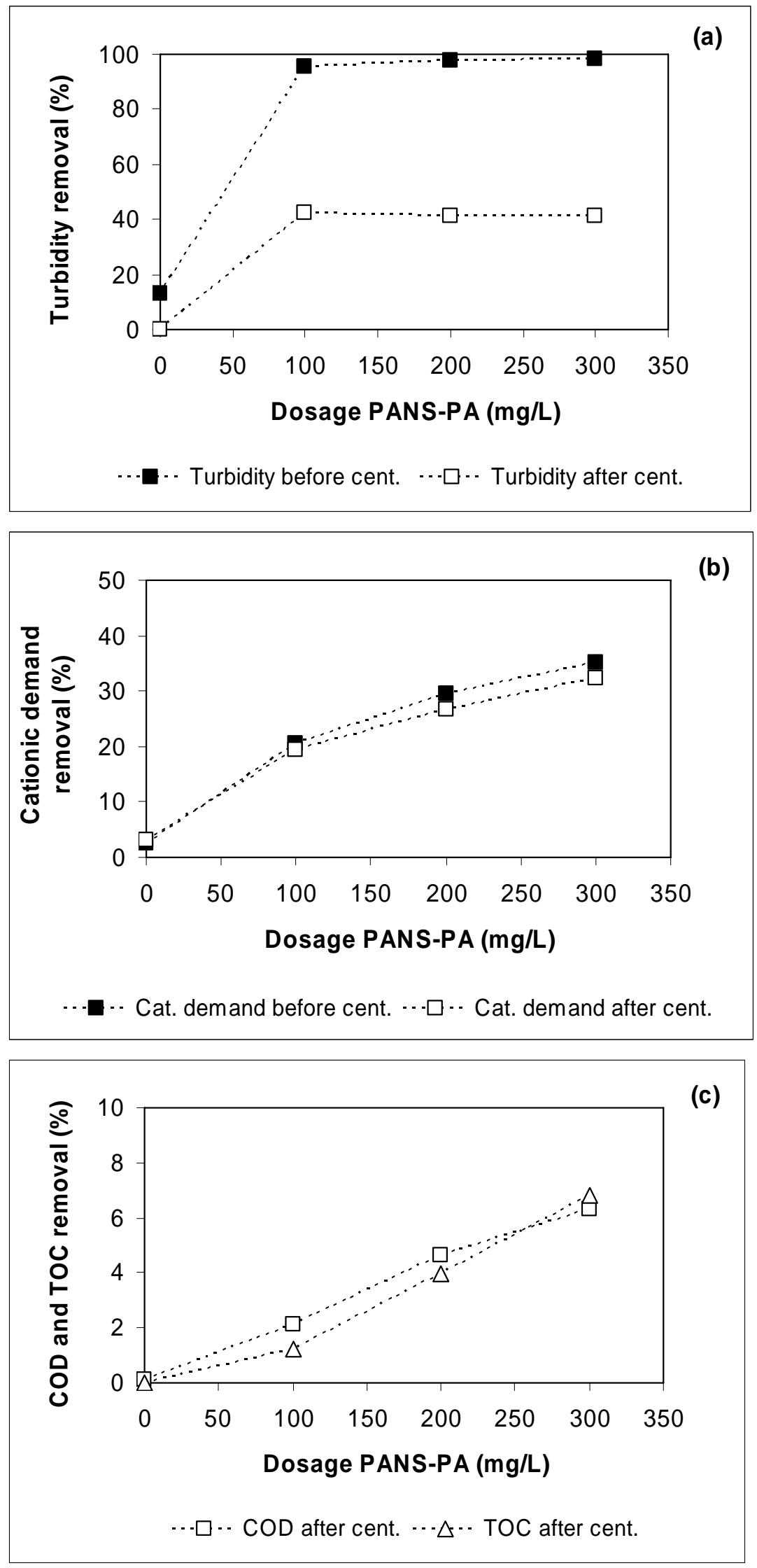

Figure 4. Removal of contaminants during the short trial: (a) Turbidity; (b) Cationic demand; (c) COD and TOC. 
In Part 1 of this work, lab trials with waters from LWC paper production were discussed. In that case, the feed waters have a similar conductivity and COD than those during the one-day short trial but with a higher turbidity (12200 NTU) and lower cationic demand $(0.56 \mathrm{meq} / \mathrm{L})$. The removal efficiencies obtained at lab-scale, with a dosage of $300 \mathrm{mg} / \mathrm{L}$, were lower for COD (4\% removal) and turbidity (83\% removal), probably due to the higher turbidity of the inlet waters, and slightly better for cationic demand (40\%), probably due to the lower cationic demand of the inlet waters during lab trials. This comparison shows that the industrial efficiency of DAF units can be reasonably predicted by the methodology followed at lab-scale.

\subsection{Long-term field trials: newsprint production}

A total of 21 samples were taken during the trials: 7 samples without the addition of any chemical (0/0), 2 samples with only an addition of coagulant (130/0), 4 samples with only an addition of flocculant $(0 / 3)$ and 8 samples using dual systems $(70 / 3,80 / 3$, $100 / 3,100 / 3,110 / 3,110 / 3,130 / 3$ and 230/3).

The main results in terms of turbidity, cationic demand and COD removal are summarized in Figure 5. In newsprint production, just DAF (without the addition of any chemical) removed nearly $70 \%$ of the turbidity of the inlet waters (average of 7 samples). The addition of $3 \mathrm{ppm}$ of flocculant improved this removal efficiency to more than $80 \%$ (average of 4 samples). Compared to not using chemicals or only flocculant, the addition of only coagulant does not improve the efficiency in turbidity removal. When PANS-PA and flocculant are used in conjunction, at dosages of $3 \mathrm{ppm}$ of flocculant and variable dosages of PANS-PA $(70-230 \mathrm{ppm})$, the removal efficiency increased significantly to $97-99 \%$, obtaining clarified waters with only $20-40$ NTU of residual turbidity. The lowest dosages of PANS-PA tested (70-80 ppm) achieved similar results to that of the highest dosages (230 ppm). However, the lowest dosages of PANSPA were tested in period $\mathrm{B}$ and the end of the period D (when the contamination load of the waters was lower compared to the beginning of period D, where the highest dosages were tested) (see Table 2). The same was observed in terms of total solids removal (without figure): there is a very high reduction of solids without the use of chemicals or using only flocculant, achieving values over $98 \%$. Therefore, for turbidity and solids removal, the cost of adding a coagulant is not justified.

The situation is different with cationic demand and COD. In cationic demand, there is no reduction if chemicals are not used; the removal efficiency is around 4\% (average of 7 samples). The use of flocculant slightly improved the removal efficiency until values of around 12\% (average of 3 samples). If only coagulant is added, a significant removal of cationic demand is observed: 130 ppm of PANS-PA reduced a $56 \%$ of the cationic demand (average of two samples). However, the combination of coagulant and flocculant is necessary for achieving higher reductions; depending on the dosage and the level of contamination load of the waters, cationic demand is reduced from 70 up to $90 \%$. Higher removal efficiencies are obtained in period B than in period D. Dosages as 
low as $70 / 3$ or $80 / 3$ reduced $80-90 \%$ of the cationic demand of the waters in regular conditions $(300-400 \mu \mathrm{eq} / \mathrm{L})$. If the inlet cationic demand is higher, a higher quantity of coagulant is needed to achieve a similar removal (over $200 \mathrm{mg} / \mathrm{L}$ ). Removals of cationic demand can vary depending on the system studied, but removals higher than 40-60\% are important and rarely observed in the literature ${ }^{10}$.

On the other hand, COD removal depends very much on the value of the COD of the inlet waters. The lower the inlet COD, the higher the COD removal, although these reductions do not go beyond $16 \%$ and the average reduction is around $8 \%$. It is clearly demonstrated that if COD removal is required, coagulant addition is needed.

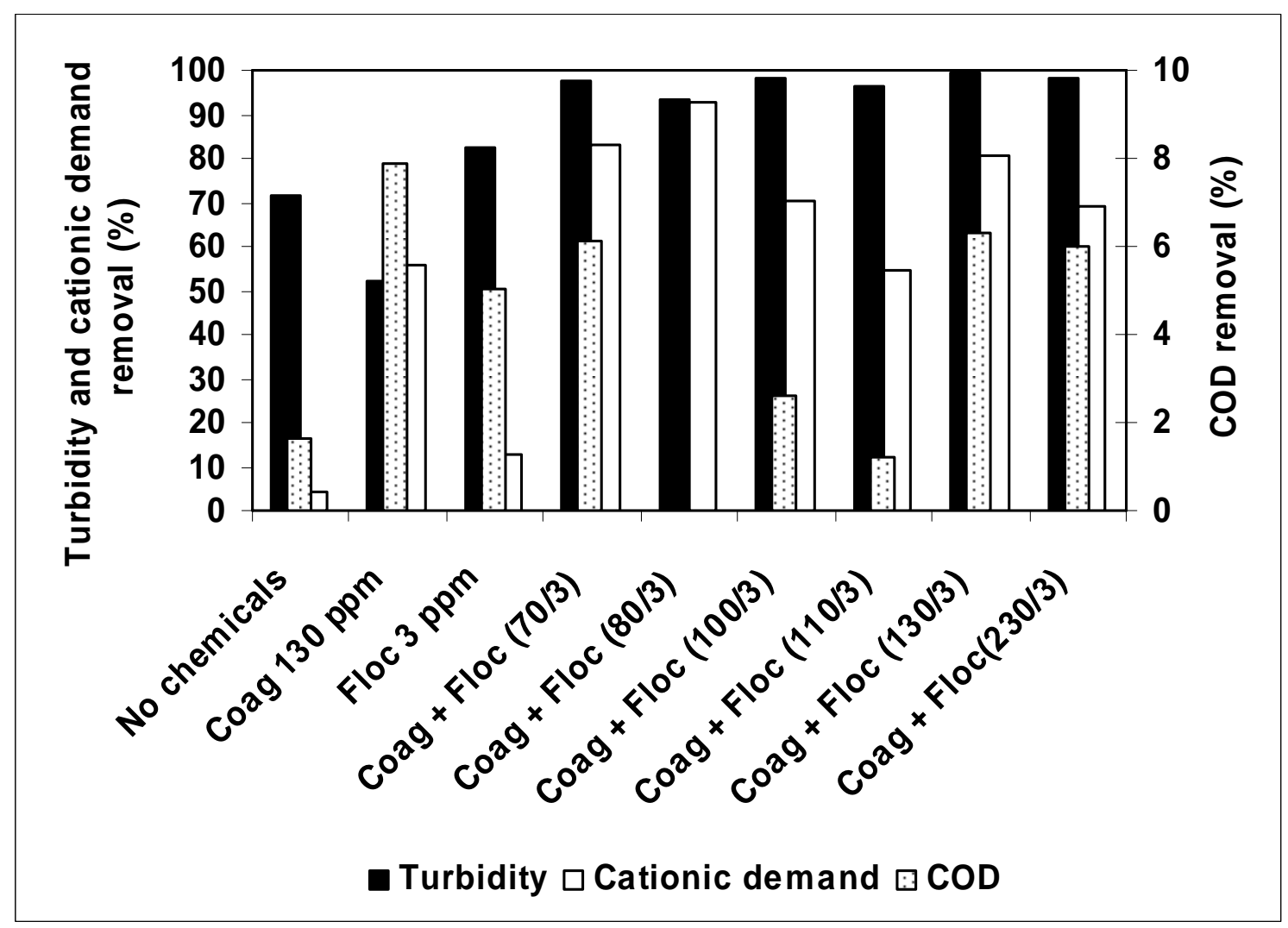

Figure 5. Long-term field trials. Newsprint production.

In newsprint production, microstickies and secondary stickies were only reduced by $16 \%$ and $12 \%$, respectively, at industrial conditions if only flocculant is added $(3 \mathrm{mg} / \mathrm{L})$. When the addition of PANS-PA started in conjunction with the flocculant (70/3), the removal of microstickies increased to $42 \%$ and the removal of secondary stickies to $75 \%$. Good performance of the aluminium chemicals could be explained by the fact that microstickies and potential secondary stickies are negatively charged and are stabilized by the presence of an electrical double layer which can be destabilized by neutralization with electrolytes such as $\mathrm{Al}^{3+}$, as $\mathrm{X}$. Huo et al. reported in the analysis of model microstickies ${ }^{11}$.

\subsection{Long-term field trials: LWC paper production}


A total of 9 samples were taken during the trials: 7 samples in stage $C(0 / 3,70 / 3$, $130 / 3,170 / 3,270 / 3$ and 300/3) and 2 samples in stage $\mathrm{E}(0 / 0$ and $0 / 3)$. In this case, the contamination load of the feed waters is very similar (see Table 2 ) and the results can be compared directly although samples were taken from different stages.

The contamination load of waters from LWC paper production is around three times higher than from newsprint production in terms of conductivity and COD and around six times higher in terms of cationic demand and turbidity. Regarding the characteristics of the contaminants, waters from LWC paper production have a higher number of particles (Figure 6) and with a smaller size (Figure 7), making their removal by DAF more difficult. Furthermore, in the case of LWC paper, $82.4 \%$ of the particles are smaller than $10 \mu \mathrm{m}$ while in newsprint this value is $59.3 \%$. As it was stated in Part 1 of this work, particles smaller than $1 \mu \mathrm{m}$ are present but are not detected by the FBRM technique.

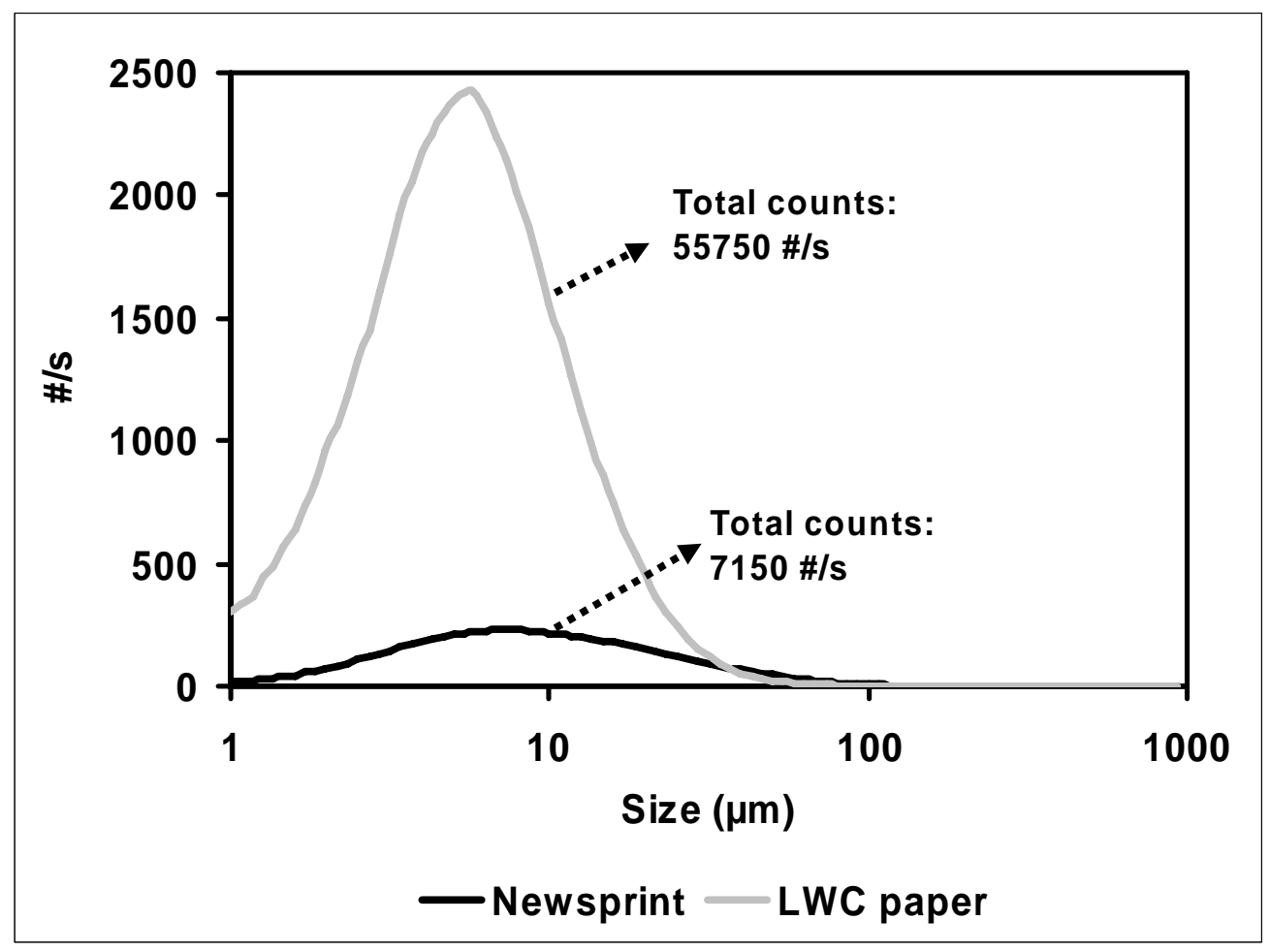

Figure 6. Comparison of the chord size distributions of the inlet waters to DAF. 


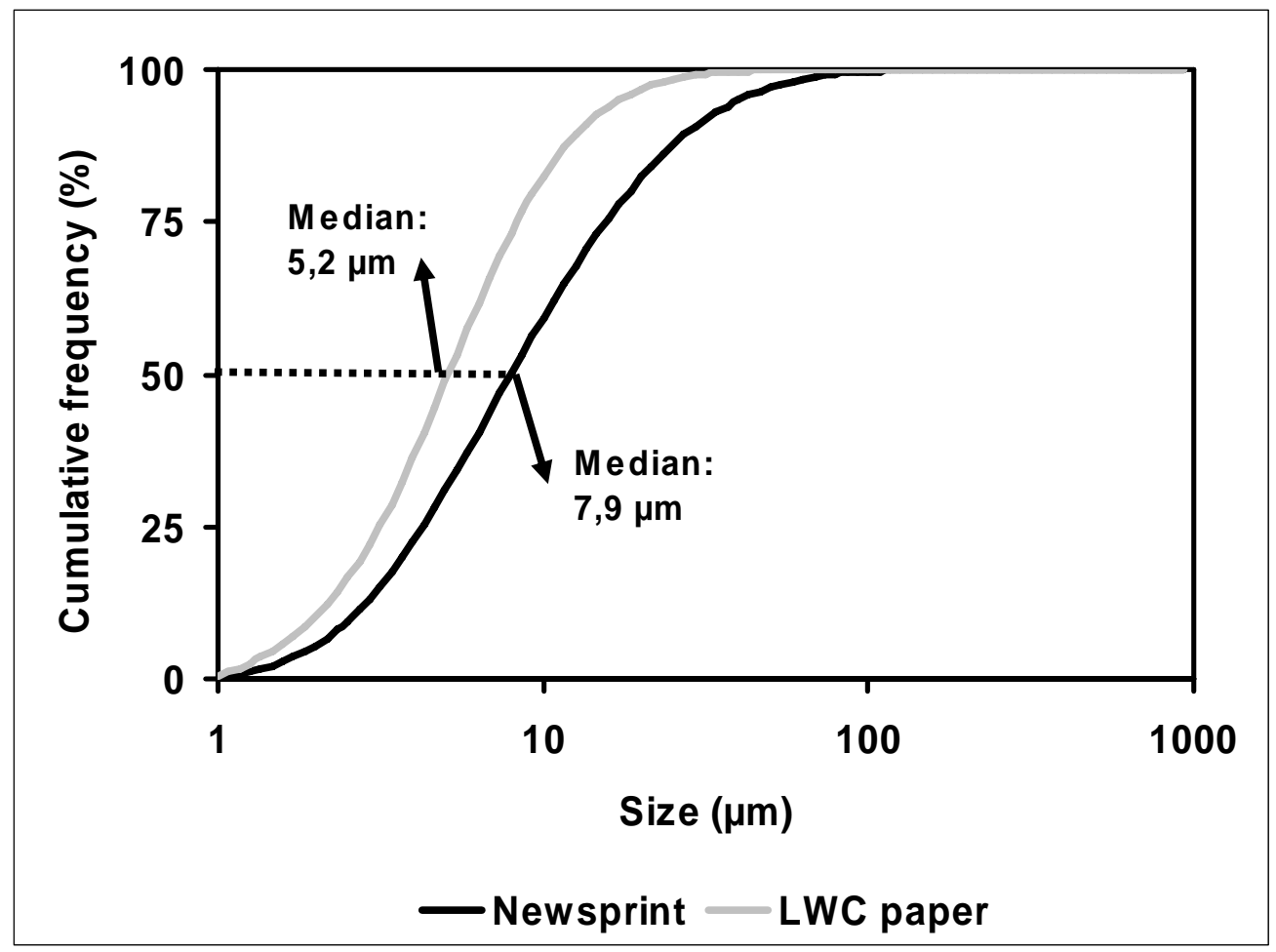

Figure 7. Comparison of the cumulative frequency curve of the chord size of the inlet waters to DAF.

Main results obtained during LWC paper production are summarized in Figure 8. For this type of production, both coagulant and flocculant are needed for an optimal turbidity reduction. With no chemicals, no turbidity reduction is observed; a $25 \%$ reduction is observed using only flocculant $(0 / 3)$ but when adding only coagulant $(70 / 0)$ the removal is around 55\%. Higher dosages of coagulant are needed for obtaining high removal efficiencies: removals in turbidity from $75 \%$ with a $70 / 3$ treatment to more than $99 \%$ can be obtained if 300/3 treatment is used (residual turbidity of only 100-200 NTU). The trend observed for solids is similar to that obtained for turbidity (not shown). For an optimal solids removal, high dosages of PANS-PA are needed (over 250 $\mathrm{mg} / \mathrm{L}$ ). Flocculant addition only removed around $55 \%$ of total solids while different combinations of PANS-PA and flocculant improved the removal efficiency to more than $95 \%(270 / 3)$ and $99 \%(300 / 3)$.

There is no reduction of cationic demand if chemicals are not used or if only flocculant is added (removal $<4 \%$ ). With the addition of only coagulant, the removal efficiency is noticeable: $10 \%$ using a $70 / 0$ treatment. The cationic demand removal efficiency increases with higher dosages of PANS-PA, achieving an approximate $45 \%$ reduction with the highest dosage (300/3). For similar dosages of coagulant, the cationic demand removals are around half of those obtained with waters from newsprint production due to the higher cationic demand of the inlet waters. Similar results were obtained for COD, without chemicals or only flocculant, with a reduction of nearly $0 \%$ 
and 3\%, respectively. Only when PANS-PA was used could the COD reduction be increased until $7 \%$, reached at the highest dosages (300/3).

In LWC production, due to problems in the feeding system of PANS-PA during the trials, samples from the inlet of DAF were taken and PANS-PA was added at lab scale for determining secondary or potential stickies. In this case, high concentrations were necessary (around $500 \mathrm{mg} / \mathrm{L}$ ) for the observation of the effect on the water. A $34 \%$ removal in secondary stickies was measured. Removal of secondary stickies is relatively low compared to the waters from newsprint paper production due to the higher contamination load of the waters. Microstickies were not measured.

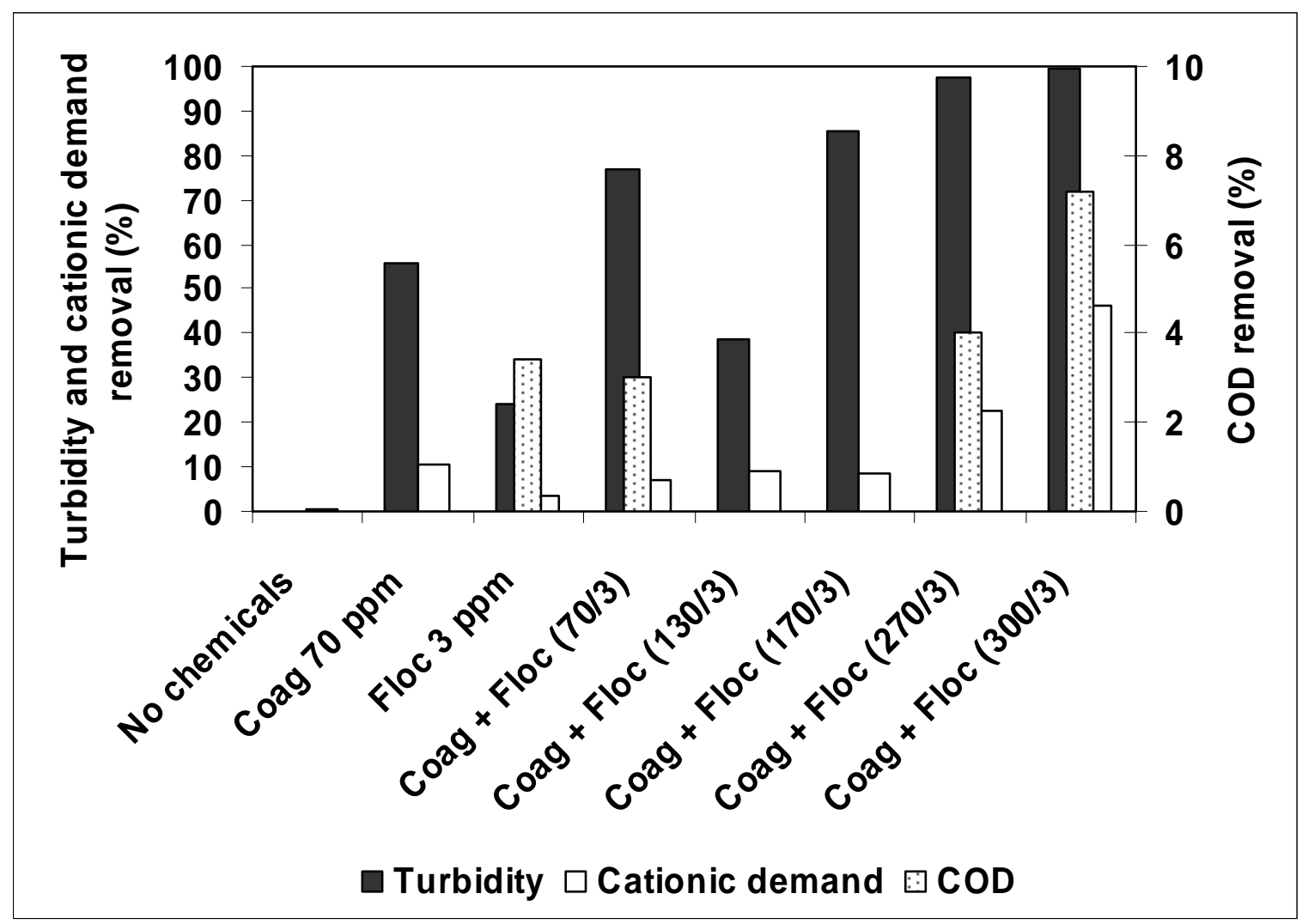

Figure 8. Long-term field trials with PANS-PA. LWC production.

\subsection{Economical consideration}

The comparison of the costs for the different DAF treatments is difficult because first, the new chemical has not yet been commercialized and second, the chemical suppliers may vary the price of a product according to their commercial strategy. In this case, it is estimated that the price of the new product could be similar to a coagulant price, which means it would be much cheaper than a traditional flocculant.

However, the main benefits in this case would be based in the possibility of increasing the removal of contaminants from process waters, especially DCM which will allow a further closure of the water circuits and a lower fresh water consumption 
whose price is increasing continuously. This is especially important for LWC paper production.

\section{CONCLUSIONS}

Part 1 of this work allowed us to select a combination of a polyaluminium nitrate sulphate and a cationic polyamine as the most promising chemical for the removal of contaminants by DAF.

Field trials have showed that the benefits of the substitution of currently used chemicals at the mill by PANS-PA in newsprint production, according to turbidity and total solids, is not critical. The addition of only flocculant (3 ppm) is enough for obtaining high removal efficiencies of around $80 \%$. If higher removal efficiencies are necessary, low dosages of PANS-PA (70-80 ppm) could increase the removal efficiency of turbidity to $97-99 \%$. The opposite is true for the case of cationic demand and COD. If only flocculant is added, no significant removal of cationic demand and COD are obtained. If a good removal of cationic demand is needed, it is necessary to add PANSPA. The coagulant dosage depends on the cationic demand of the inlet water. In the case of COD reduction, it is not possible to increase the reduction over $15 \%$ even with high dosages. The use of PANS-PA reduced microstickies and secondary stickies very efficiently, by $42 \%$ and $75 \%$, respectively.

In the case of LWC production, the use of PANS-PA is completely necessary for obtaining the removal of contaminants in the DAF unit due to the higher contamination load of the circuits. In this case, higher dosages are needed compared to newsprint production, not only for the removal of cationic demand and COD, but also for turbidity removal. Even with the highest dosages tested, it is not possible to achieve the same removal efficiencies obtained for newsprint production. The optimal dosage would be between 200 and $300 \mathrm{mg} / \mathrm{L}$ of coagulant. These dosages could reduce turbidity from 80 to $99 \%$ and cationic demand from 10 to $45 \%$. Even with the highest dosage tested (300 $\mathrm{mg} / \mathrm{L}$ ), COD reduction is low (around 7\%). A reduction of $34 \%$ in secondary stickies was achieved at high dosages.

The industrial efficiency of DAF units has been reasonably predicted by the lab-scale methodology used.

\section{ACKNOWLEDGMENTS}

Authors thank the financial support from the European Union through Project "Reduction of detrimental substances in papermaking" (QLK5-CT-2001-00749) and from the Madrid Community through the program PROLIPAPEL (S-0505/AMB/0100). The collaboration of Holmen Paper Madrid and Sachtleben Chemie GmbH are also acknowledged. 


\section{REFERENCES}

(1) Miranda, R.; Balea, A.; Sanchez de la Blanca, E.; Carrillo, I.; Blanco, A. Identification of recalcitrant stickies and their sources in newsprint production. Ind. Eng. Chem. Res. 2008, 47, 6239-6250.

(2) Miranda, R.; Blanco, A.; Negro, C. Accumulation of dissolved and colloidal material in papermaking - application to simulation. Chem. Eng. J. 2008, doi:10.1016/j.cej.2008.09.014.

(3) Saarimaa, V.; Sundberg, A.; Holmbom, B. H.; Blanco, A.; Negro, C.; Fuente, E. Purification of peroxide-bleached TMP water by dissolved air flotation. Tappi J. 2006, 5,15 .

(4) Saarimaa, V.; Sundberg, A.; Holmbom, B.; Blanco, A.; Fuente, E.; Negro, C. Monitoring of dissolved air flotation by focused beam reflectance measurement. Ind. Eng. Chem. Res. 2006, 45, 7256.

(5) Miranda, R.; Blanco, A.; de la Fuente, E.; Negro, C. Separation of contaminants from deinking process water by dissolved air flotation: effect of flocculant charge density. Sep. Sci. Technol. 2008, 43, 3732.

(6) Miranda, R.; Negro, C.; Blanco, A. Internal treatment of process waters in paper production by dissolved air flotation with new developed chemicals. Part 1: Laboratory tests. Ind. Eng. Chem. Res. 2008 (accepted).

(7) Pernitsky, D. J.; Edzwald, J. K. Selection of alum and polyaluminum coagulants: principles and applications. J. Water Supply Res. Technol.-Aqua 2006, 55, 121

(8) Monte, M. C.; Blanco, A.; Negro, C.; Tijero, J. Development of a methodology to predict sticky deposits due to the destabilisation of dissolved and colloidal material in papermaking - application to different systems. Chem. Eng. J. 2004, 105, 21.

(9) Blanco, A.; Miranda, R.; Negro, C.; Garcia-Suarez, C.; Garcia-Prol, M.; Sanchez, A. Full characterization of stickies in a newsprint mill: the need for a complementary approach. Tappi J. 2007, 6, 19.

(10) Roring, A.; Wackerberg, E. Characterization of deinking white water-influence on flotation and bleaching efficiency. Pulp Pap. Can. 1997, 98, 17.

(11) Huo, X.; Venditti, R.A.; Chang, H.-M. Effect of cationic polymers, salts and fibres on the stability of model micro-stickies. J. Pulp Pap. Sci. 2001, 27, 207. 